EPSC Abstracts

Vol. 15, EPSC2021-678, 2021

https://doi.org/10.5194/epsc2021-678

Europlanet Science Congress 2021

(C) Author(s) 2021. This work is distributed under

the Creative Commons Attribution 4.0 License.

\title{
Detecting Venus' volcanic gas plumes with VenSpec-H
}

\author{
Séverine Robert ${ }^{1,2}$, Caterina Macovenco ${ }^{1,3}$, Maxence Lefèvre ${ }^{4}$, Colin Wilson ${ }^{4}$, Emmanuel Marcq ${ }^{5}$, \\ Bruno Bézard $^{6}$, Jörn Helbert ${ }^{7}$, and Ann Carine Vandaele ${ }^{1}$ \\ ${ }^{1}$ Royal Belgian Institute for Space Aeronomy, Brussels, Belgium (severine.robert@aeronomie.be) \\ ${ }^{2}$ Institute of Condensed Matter and Nanosciences, Université catholique de Louvain, Belgium \\ ${ }^{3}$ Ecole Centrale de Lille, France \\ ${ }^{4}$ Dept. of Physics, Oxford University, Oxford, UK \\ ${ }^{5}$ LATMOS, Université de Versailles Saint-Quentin, France \\ ${ }^{6}$ LESIA, Observatoire de Paris, Meudon, France \\ ${ }^{7}$ German Aerospace Center, DLR, Germany
}

The VenSpec-H instrument is part of the EnVision payload which is currently being evaluated by ESA for mission selection. EnVision is a medium class mission to determine the nature and current state of geological activity on Venus, and its relationship with the atmosphere, to understand how Venus and Earth could have evolved so differently.

VenSpec-H is part of the VenSpec suite [1], including also an IR mapper and a UV spectrometer [2] suite. The science objectives of this suite are to search for temporal variations in surface temperatures and tropospheric concentrations of volcanically emitted gases, indicative of volcanic eruptions; and study surface-atmosphere interactions and weathering by mapping surface emissivity and tropospheric gas abundances. Recent and perhaps ongoing volcanic activity has been inferred in data from both Venus Express and Magellan. Maintenance of the clouds requires a constant input of $\mathrm{H}_{2} \mathrm{O}$ and $\mathrm{SO}_{2}$. A large eruption would locally alter the composition by increasing abundances of $\mathrm{H}_{2} \mathrm{O}$, $\mathrm{SO}_{2}$ and $\mathrm{CO}$ and perhaps decreasing $\mathrm{D} / \mathrm{H}$ ratio. Observations of changes in lower atmospheric $\mathrm{SO}_{2}$, $\mathrm{CO}$ and $\mathrm{H}_{2} \mathrm{O}$ vapour levels, cloud level $\mathrm{H}_{2} \mathrm{SO}_{4}$ droplet concentration, and mesospheric $\mathrm{SO}_{2}$, are therefore required to link specific volcanic events with past and ongoing observations of the variable and dynamic mesosphere, to understand both the importance of volatiles in volcanic activity on Venus and their effect on cloud maintenance and dynamics.

To contribute to this investigation, VenSpec- $\mathrm{H}$ is designed to measure $\mathrm{H}_{2} \mathrm{O}$ and $\mathrm{HDO}$ contents in the first scale height of Venus' atmosphere and probe $\mathrm{H}_{2} \mathrm{O}, \mathrm{HDO}, \mathrm{CO}, \mathrm{OCS}, \mathrm{SO}_{2}$ in the 30 to $40 \mathrm{~km}$ altitude range [3-8]. To assess the performances of our instrument at detecting volcanic eruptions, we defined a couple of scenarios of plume releases and simulated the corresponding spectra.

To simulate the vertical transport of the plume and the horizontal advection by the dynamics, the LMD Venus Mesoscale Model [9] is used, based on the WRF dynamical core and the Venus IPSL radiative transfer. The domain is focused on Imdr Regio, where VIRTIS observed a hotspot anomaly possibly linked to volcanic activity [10]. Tracers were added to the model representing $\mathrm{H}_{2} \mathrm{O}, \mathrm{CO}$ and $\mathrm{SO}_{2}$. The chemistry and photodissociation sources and sinks are modelled by a linear relaxation of the tracer abundance toward a value representative of the deep atmosphere with a characteristic time. The deep atmosphere abundance is set to $130 \mathrm{ppm}$ for $\mathrm{SO}_{2}, 30 \mathrm{ppm}$ for $\mathrm{H}_{2} \mathrm{O}$ and 20 ppm for 
CO. The relaxation time is set to 100 years for $\mathrm{SO}_{2}$, to 1000 years for $\mathrm{CO}$ and to 1 week, 1 month and 1 year for $\mathrm{H}_{2} \mathrm{O}$ representing the uncertainty of the chemistry timescale in that region. Several configurations are considered for the plume, an idealised set-up where the elevation height is fixed and the outgassing abundance is constant inside the plume and in time, and a more realistic set-up where a temperature and outgassing anomalies at the surface are prescribed is ongoing testing.

The radiances of the nightside atmosphere of Venus originate from the thermal emission of the surface and atmosphere. The spectra were simulated from 1 to 2.5 microns, using ASIMUT-ALVL, a line-by-line radiative transfer code developed at BIRA-IASB [11]. $\mathrm{CO}_{2}, \mathrm{H}_{2} \mathrm{O}, \mathrm{HDO}, \mathrm{CO}, \mathrm{SO}_{2}$, and OCS, as well as aerosols were included.

The performances of the instrument will be described in terms of its capabilities to detect small variations in the atmosphere.

\section{References}

[1] J. Helbert et al., "The VenSpec suite on the ESA EnVision mission to Venus", Proc. SPIE 11128, Infrared Remote Sensing and Instrumentation XXVII, 1112804 (2019).

[2] E. Marcq et al., "Instrumental requirements for the study of Venus' cloud top using the UV imaging spectrometer VeSUV", Advances in Space Research, 68 (2021) 275-291.

[3] B. Bézard et al., "Water vapor abundance near the surface of Venus from Venus Express/VIRTIS observations," Journal of Geophysical Research, Planets, 114 (2009) E00B39.

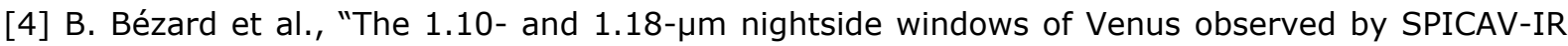
aboard Venus Express," Icarus, 216 (2011) 173-183.

[5] A. Fedorova et al., "The $\mathrm{CO}_{2}$ continuum absorption in the 1.10-and 1.18- $\mathrm{um}$ windows on Venus from Maxwell Montes transits by SPICAV IR onboard Venus Express," Planetary and Space Science, 113 (2015) 66-77.

[6] E. Marcq et al., "Remote sensing of Venus' lower atmosphere from ground-based IR spectroscopy: latitudinal and vertical distribution of minor species," Planetary and Space Science, 54 (2006) 1360-1370.

[7] E. Marcq et al., "A latitudinal survey of $\mathrm{CO}$, OCS, $\mathrm{H}_{2} \mathrm{O}$, and $\mathrm{SO}_{2}$ in the lower atmosphere of Venus: spectroscopic studies using VIRTIS-H," Journal of Geophysical Research, Planets, 113 (2008) E00B07.

[8] E. Marcq et al., "Evidence for $\mathrm{SO}_{2}$ latitudinal variations below the clouds of Venus", Astronomy \& Astrophysics, 648 (2021) L8.

[9] M. Lefèvre, A. Spiga and S. Lebonnois, "Mesoscale modeling of Venus' bow-shape waves", Icarus, 335 (2019) 113376.

[10] E. Smrekar, et al., "Recent Hotspot Volcanism on Venus from VIRTIS Emissivity", Data. Science, 328 (2010) 605.

[11] A.C. Vandaele, M. Kruglanski and M. De Mazière, "Modeling and retrieval of atmospheric spectra using ASIMUT", Proc. of the First 'Atmospheric Science Conference', ESRIN, Frascati, Italy, 2006. 